\title{
Fatal Invasive Gastrointestinal Fungal Infection in Three Non-immunocompromised Patients
}

\author{
Zubair Ahmad1, Sidra Arshad1, Romana Idrees1, Jamshid Abdul-Ghafar² and Nasir Ud Din1
}

\begin{abstract}
The importance and frequency of invasive fungal infections in different organs, including the gastrointestinal tract, has increased due to excessive use of aggressive immunosuppressive and immunomodulatory drugs in malignant diseases and organ transplantations as well as increased infections with the human immunodeficiency virus (HIV). Fungal infections in the small intestine or colon are usually part of a disseminated disease process and reach these organs through the bloodstream. Two of the patients were infants 10 and 15 days of age, while one was 23 years old. Patients presented with abdominal pain, intestinal obstruction, and rectal bleeding. Hirchsprung disease was suspected in the two infants. In the adult patient, the diagnosis was unclear. Mucormycosis was diagnosed in two out of three cases. It is imperative for clinicians to consider invasive mycosis in the differential diagnosis in patients of all ages with treatment-resistant severe abdominal pain, diarrhea and fever.
\end{abstract}

Key Words: Digestive system, Fungi, Mucormycosis, Invasive gastroenteritis.

\section{INTRODUCTION}

The importance and frequency of invasive fungal infections in the digestive (gastrointestinal) tract has increased due to excessive use of immunomodulatory drugs in malignant diseases and organ transplantation as well as increased infections with the human immunodeficiency virus (HIV). Although fungal infections principally occur in the immunocompromised and severely ill patients, but these have been described in immunocompetent persons as well. ${ }^{1}$ These infections can affect the small as well as large intestine, can result in intestinal obstruction and even masquerade as mass lesions, mimicking colorectal carcinoma. ${ }^{2}$

Herein, we present three cases of invasive fungal infections involving the small and large intestine.

\section{CASE REPORT}

Case 1: A 23-year male presented to a private hospital in Karachi with history of abdominal pain and cramps. On physical examination, a tender mass was felt in the right iliac fossa. Colonoscopy was performed but the biopsy report was inconclusive. Colonoscopy was attempted again as patient's symptoms worsened; but

1 Department of Pathology and Laboratory Medicine, The Aga Khan University Hospital, Karachi, Pakistan

2 Department of Pathology and Laboratory Medicine, French Medical Institute for Mothers Children (FMIC), Kabul, Afghanistan

Correspondence: Dr. Sidra Arshad, Section of Histopathology, Department of Pathology and Laboratory Medicine, The Aga Khan University Hospital, Stadium Road, Karachi, Pakistan E-mail: sidra.arshad@aku.edu

Received: January 10, 2018; Accepted: September 13, 2018 failed due to unspecified reasons. Exploratory laparotomy was then performed at the same hospital.

The surgical specimen was sent to our laboratory in formalin for histological examination. It was coded as cecum with omentum and comprised of part of terminal ileum, cecum with appendix and part of ascending colon. The segment of terminal ileum measured $19.5 \times 4.0 \mathrm{~cm}$, while the cecum and ascending colon together measured $20 \times 4.5 \mathrm{~cm}$. A thickened, greyish black area measuring $14.0 \times 8.0 \times 2.5 \mathrm{~cm}$, which was suggestive of gangrene, was identified in the cecum and proximal part of ascending colon. Separately lying adipose tissue, labelled as "omentum" measured $8.0 \times 6.0 \mathrm{~cm}$, was also received. The appendix measured $5.5 \times 1.5 \mathrm{~cm}$ and wall thickness was $0.5 \mathrm{~cm}$. On opening, the lumen of the bowel as well as that of appendix was filled with fibrinopurulent exudate. Representative sections were submitted for histopathological examination, which showed severe transmural acute and chronic inflammation extending upto the serosa. Areas of necrosis were also seen. Numerous broad ribbon like, non-septate fungal hyphae were seen in the gangrenous bowel wall and appendix. These were highlighted on special stain PAS+/- and were morphologically consistent with Mucormycosis (Figure $1 \mathrm{~A}-\mathrm{C}$ ).

The diagnosis was communicated and intravenous Amphotericin B was started at the private hospital. However, the condition of the patient deteriorated and he developed abdominal distension. A relook laparotomy was performed at the private hospital for postoperative intestinal obstruction. The next day he was rushed to the Emergency of the Aga Khan University Hospital (AKUH) with signs and symptoms of acute peritonitis. He underwent an emergency exploratory laparotomy during which necrotic distal small bowel and unhealthy 


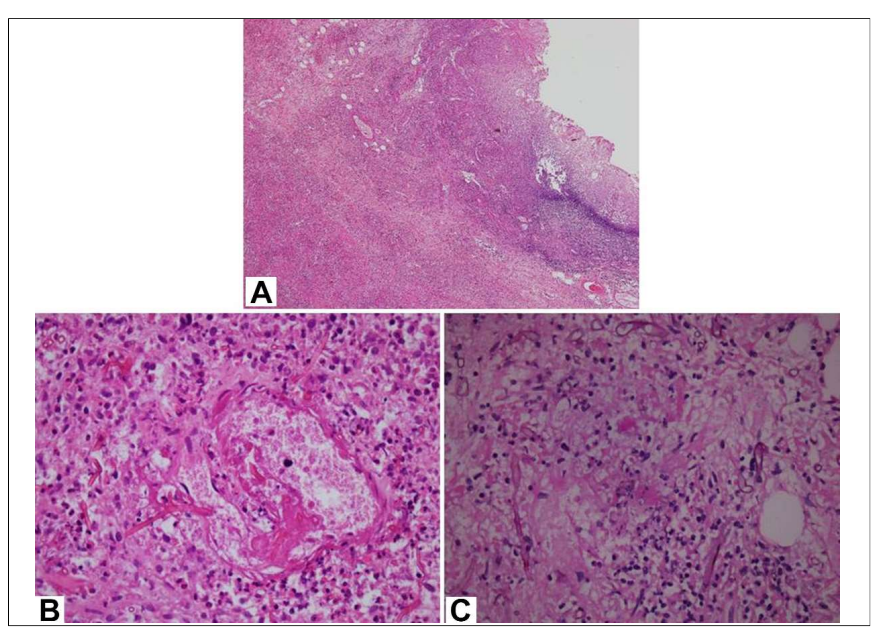

Figure 1: Small bowel wall exhibiting transmural inflammation and mucosal ulceration (A). Aseptate fungal hyphae invading a vessel wall seen in the bowel wall (B). Broad non-septate hyphae highlighted on PASD stain (C).

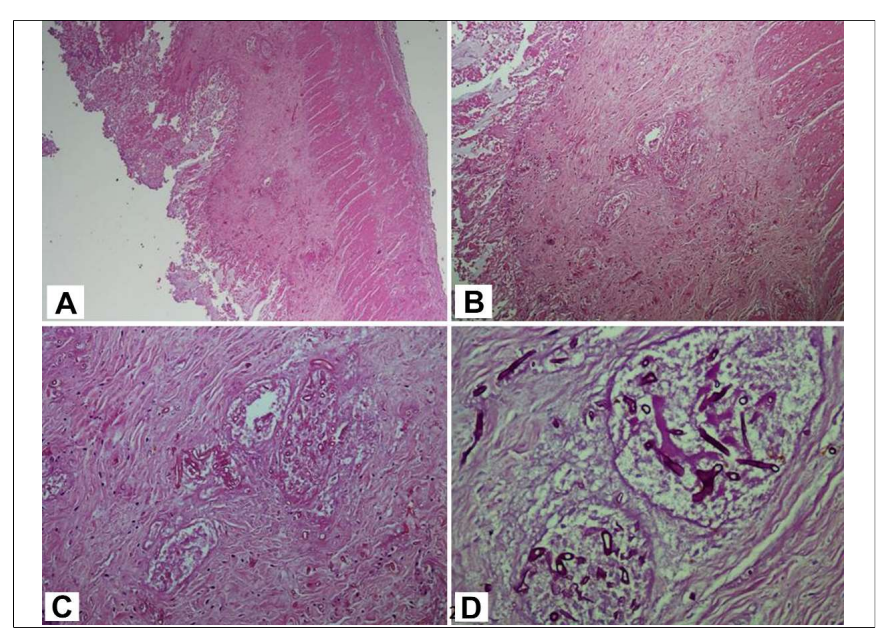

Figure 2: Appendix wall exhibiting mucosal ulceration (A). Dilated vessels in the submucosa showing hyphae of fungal organisms which are highlighted on PASD special stain (B, C, D).

mesentery were found. These were resected, and he was restarted on intravenous Amphotericin B plus intravenous broad spectrum antibacterial agents.

The specimen was received in the section coded as "necrotic distal small bowel and unhealthy mesentery" and consisted of a long segment of bowel measuring $60 \times 7.0 \mathrm{~cm}$, which was thinned out, necrotic and blackened. On opening, the mucosa appeared flattened. Representative sections submitted for histopathological examination showed similar picture as before. The patient's treatment with intravenous antibacterial agents and antifungal agent Amphotericin B continued along with surgical resection to remove the infected tissue. However, patient's condition continued to deteriorate.

He was yet again rushed to the operating room, laparotomy showed that the gangrene had by this time spread to the mesentery and the left colon, down to sigmoid and rectum. Necrotic material was seen in the retroperitoneum. Part of left colon measuring $20 \times 5.0 \mathrm{~cm}$ was resected and was received in our section. On histological examination, marked acute and chronic inflammation, necrosis and non-septate fungal hyphae were seen. Diagnosis of gangrenous left colon with severe acute and chronic inflammation compatible with invasive mucormycosis was given. Culture studies of necrotic material, sent during multiple laparotomies, revealed heavy growth of Escherichia coli and Enterococcus species alongwith moderate growth of Proteus mirabilis, while fungal culture showed growth of Candida tropicalis and Mucormycosis. Inspite of intense intravenous antibacterial and antifungal treatment and repeated surgeries to remove the infected and necrotic tissue, the unfortunate patient continued to deteriorate and died one day after the last laparotomy was performed. Consent was obtained from father of the patient for case report.

Case 2: A 10-day male infant did not pass stool from the time of his birth. He remained in the private hospital where he was delivered and was operated for suspected intestinal obstruction. The authors received a rectal biopsy with clinical suspicion of Hirschsprung disease. It consisted of a single small piece of grey white tissue measuring $0.2 \times 0.1 \mathrm{~cm}$. In a separate container labelled as "cecum and appendix", we received a segment of cecum with attached appendix. The bowel segment measured $3.0 \times 1.5 \mathrm{~cm}$ in dimension. The attached appendix measured $2.0 \times 0.7 \mathrm{~cm}$. On opening the bowel segment, the mucosa appeared unremarkable. Representative sections from cecum and appendix were submitted for microscopic examination. The rectal biopsy was entirely submitted for the same. The requisition form signed by the clinician asked the pathologist to look for ganglion cells especially and any hypertrophic nerve bundles. The rectal biopsy did not show any significant histological abnormality; and ganglion cells were present in the submucosa and the muscle wall. Microscopic sections from the cecum and appendix also demonstrated the presence of ganglion cells. However, the wall showed severe acute and chronic inflammation and areas of necrosis. A number of broad, non-septate fungal hyphae were seen which were morphologically consistent with mucormycosis (Figures 2 A-D).

Report was urgently communicated to the clinician. Intravenous antifungal and antibacterial treatment was started but the infant's condition worsened and he died on the fifteenth day of life. Consent was obtained from father of the patient for case report.

Case 3: A 15-day male infant with a history of two episodes of bleeding from mouth and one episode of bleeding per rectum was noticed to have a swelling (lump) in the right iliac region. He also had repeated episodes of fever. Due to repeated episodes of bleeding, he remained admitted in hospital from the time of birth. The local surgeons who examined the baby boy 
suspected Hirschsprung disease/appendicular lump and performed exploratory laparotomy with appendectomy and ileostomy.

The specimens were submitted to the Department for histological evaluation and were received in two separate containers. The first container was coded as 'Colon' and consisted of a segment of bowel wall measuring $1.0 \times 1.0 \times 0.8 \mathrm{~cm}$ in dimension. The second container was coded as "Appendix" and consisted of an intact appendix measuring $3.4 \times 0.3 \mathrm{~cm}$. On sectioning the appendix, lumen measured $0.1 \mathrm{~cm}$ in diameter while average wall thickness was $0.2 \mathrm{~cm}$. Both specimens were entirely submitted for histological examination. Microscopic examination of sections from both colon and appendix revealed mucosal ulceration and severe acute and chronic transmural inflammation with extensive involvement of the serosa. Areas of necrotic exudate were seen on the outer surface. Both the specimens showed numerous septate fungal hyphae with branching at right angles. Light microscopic appearance was compatible with aspergillus species.

Diagnosis was communicated urgently to the clinician. However, the baby could not survive and died two days after the diagnosis. Consent was obtained from father of the patient for case report.

\section{DISCUSSION}

In 1992, Prescott et al. reported 14 cases of invasive fungal infections of the small and large intestine in patients with carcinoma of the intestine, who were immunosuppressed because of immunosuppressive therapy. ${ }^{3}$ All 14 cases demonstrated either Candida or Aspergillus species or both combined. These invasive mycotic infections grossly produce ulcers, sloughed mucosal membranes and polypoid masses. Candida organisms usually caused mucosal invasion while Aspergillus organisms were associated with transmural invasion. Eggimann et al. reported two cases of invasive Aspergillus of small intestine. Both patients were immunosuppressed as a result of treatment given for acute myeloid leukemia. Both cases showed transmural involvement and vascular invasion. One of the two patients died just over two weeks after admission in spite of extensive support and amphotericin B therapy. The Authors reviewed a large number of series both retrospective and prospective. According to the data reviewed by Eggimann et al. diagnosis before death was made in $14-56 \%$ cases in autopsy series, $28-100 \%$ cases in retrospective series and $99-100 \%$ cases in prospective series. Their detailed review also revealed that mortality rates were $27-88 \%$ in retrospective series and $32-64 \%$ in various prospective series indicating that patients included in prospective studies were either less critically ill or received early antifungal treatment. ${ }^{4}$ Of the present three patients, Aspergillus species were found in the 15-day baby who had no definite risk factors and was not immunosuppressed. However, the mother had history of chorioamnionitis and delivery was performed at a health centre in their village. A recent case report by Cha et al. reported an invasive colonic Aspergillus in a 47-year-old immunocompetent male who did not have the classic risk factors for development of invasive Aspergillosis. The patient was diabetic and they concluded that critically ill patients, prolonged stay in intensive care units (ICUs), prolonged antibiotic use, mechanical ventilation etc. may be some of the nonclassical risk factors for invasive aspergillosis of the intestine. 5

The second and third patients did not have any apparent risk factors for development of invasive mycosis of the colon. No obvious cause for immunosuppression was found in either of these patients. As shown by culture studies, the 23-year male not only had severe bacterial infection of the colon but also combined invasive fungal infection comprising Candida and Mucormycosis species. The third baby, a 10-day-old, had invasive mucormycosis infection. A number of recent studies have shown that paracoccidiodomycosis and histoplasmosis followed by candidiasis and cryptococcosis are the commonest fungal infections involving the colon in HIV positive/immune compromised patients with very low CD4 counts comprising over $90 \%$ of all invasive mycoses of the colon in such patients. Aspergillosis is also relatively common and may account for almost $9 \%$ of all colonic invasive mycosis. Mucormycosis is very uncommon and accounts for less than $1.0 \%$ of all invasive colonic fungal infections. However, mucormycosis has emerged as an important cause of invasive fungal infection in patients with hematologic malignancies and causes considerable morbidity and mortality in these patients. ${ }^{6}$ Yet, two out of the three patients showed invasive Mucormycosis. It must also be noted that while colonic aspergillosis and candidiasis occur almost exclusively in immunocompromised patients, a significant percentage of invasive colonic Mucormycosis and Cryptoccocosis occur in the immunocompetent. ${ }^{7}$ Prognosis is very good in gastrointestinal histoplasmosis if recognised early and treated aggressively. However, it can be fatal if left untreated. ${ }^{8}$ Studies have also shown that invasive colonic aspergillosis has a $50 \%$ treatment response to Amphotericin B.9 Some studies report a $100 \%$ treatment response to antifungal treatment with Fluconazole for invasive colonic candidiasis. However, the response rate depends upon the species and resistance to azoles. First line antifungal agents including azoles are effective but antifungal resistance has emerged in some candida species and the need to administer antifungal drugs judiciously cannot be underscored.10 Unfortunately, all three patients in our series died in spite of treatment. In all three cases, fungal infection was not suspected and specific antifungal 
treatment was not started until it was too late which led to the tragic deaths of two infants and a young man.

It must be remembered that Candida species are the main fungal strains of the gut flora but gastrointestinal tract surgery can result in mucosal disruption and dissemination of candida in the blood stream from where they can secondarily invade any organ including the gut itself and cause severe and life threatening damage with marked bowel infarction. Carlo et al. reported two cases of invasive candidiasis of the stomach and small intestine, one of which occurred following colorectal surgery for rectal carcinoma. ${ }^{9}$ Severe abdominal pain is the most common symptom in colonic aspergillosis and mucormycosis. Both these fungal infections lead to deep invasion of colonic wall as well as vascular invasion and this may explain the abdominal pain. 2,7

It is imperative for clinicians in our setting to consider invasive mycosis in the gastrointestinal tract in the differential diagnosis in patients of all ages who develop sudden severe unexplained abdominal pain, diarrhea and fever, which do not respond to treatment and whose condition appears to be deteriorating. Keeping in view the rarity of these cases, secondary fungal infections developing on top of other primary pathologies (which might have led to the disruption of the mucosal barrier and thus facilitated the development of invasive fungal infections) should also be considered. It must be noted that prognosis is still poor in patients with gastrointestinal mucormycosis and aspergillosis. The interesting finding in this series was that mucormycosis accounted for two of the three cases of invasive fungal infections. This is in contrast to the studies which demonstrate that mucormycosis accounts for less than $1 \%$ of all invasive colonic fungal infections. ${ }^{7}$

\section{REFERENCES}

1. Odze RD, Goldblum JR. Surgical pathology of the GI tract, liver, biliary tract, and pancreas. Infect Dis GI Tract 2009; 51-79.

2. Ilyas MIM, Jordan SA, Nfonsam V. Fungal inflammatory masses masquerading as colorectal cancer: A case report. BMC Res Notes 2015; 8:32.

3. Prescott R, Harris M, Banerjee SS. Fungal infections of the small and large intestine. J Clin Pathol 1992; 45:806-11.

4. Eggimann P, Chevrolet JC, Starobinski M, Majno P, Totsch M, Chapuis $\mathrm{B}$, et al. Primary invasive aspergillosis of the digestive tract: report of two cases and review of the literature. Infection 2006; 34:333-8.

5. Cha SA, Kim MH, Lim TS, Kim HH, Chang KY, Park HS, et al. Invasive primary colonic aspergillosis in the immunocompetent host without classical risk factors. Yonsei Med J 2015; 56: 1453-6.

6. Alghamdi A, Lutynski A, Minden M, Rotstein C. Successful treatment of gastrointestinal mucormycosis in an adult with acute leukemia: case report and literature review. Curr Oncol 2017; 24:61.

7. Roden MM, Zaoutis TE, Buchanan WL, Knudsen TA, Sarkisova TA, Schaufele RL, et al. Epidemiology and outcome of zygomycosis: a review of 929 reported cases. Clin Infect Dis 2005; 41:634-53.

8. Sharma R, Lipi L, Gajendra S, Mohapatra I, Goel RK, Duggal R, et al. Gastrointestinal Histoplasmosis: A Case Series. Int J Surg Pathol 2017; 25:592-8.

9. Di Carlo P, Di Vita G, Guadagnino G, Cocorullo G, D'Arpa F, Salamone $G$, et al. Surgical pathology and the diagnosis of invasive visceral yeast infection: two case reports and literature review. World J Emerg Surg 2013; 8:38.

10. Pappas PG, Lionakis MS, Arendrup MC, Ostrosky-Zeichner L, Kullberg BJ. Invasive candidiasis. Send to Nat Rev Dis Primers 2018; 4:18026. 